\title{
The CMS Level-1 tau lepton and Vector Boson Fusion triggers for the LHC Run II
}

\author{
Chiara Amendola* \\ Laboratoire Leprince-Ringuet (École polytechnique) \\ on behalf of the CMS Collaboration \\ E-mail: chiara.amendola@cern.ch
}

The CMS experiment implements a sophisticated two-level triggering system composed of a Level-1 trigger, instrumented by custom-design hardware boards, and a software High-LevelTrigger. A new Level-1 trigger architecture with improved performance is now being used to maintain thresholds compatible with the CMS physics program despite the challenging luminosity conditions experienced during Run II. The upgrade of the calorimetry trigger is presented along with performance data. The implementation of the first dedicated Vector Boson Fusion trigger algorithm is described and the performance on benchmark physics signal is assessed.

EPS-HEP 2017, European Physical Society Conference on High Energy Physics 5-12 July 2017

Venice, Italy

* Speaker. 


\section{Introduction}

The CMS (Compact Muon Solenoid) experiment, operating at the LHC (Large Hadron Collider, CERN, Switzerland), uses a multi-purpose detector designed specifically to characterise different kind of particles and it is currently collecting data corresponding to $\sqrt{s}=13 \mathrm{TeV}$ protonproton collisions. For a high-luminosity experiment such as CMS, the trigger system is essential. The rate of bunch crossing $(\sim 40 \mathrm{MHz})$ is far beyond the sustainable event output rate $(\sim 1 \mathrm{kHz})$ and the $\sim 3 \mathrm{~Gb} / \mathrm{s}$ throughput rate. The two-level CMS trigger, consisting of the Level-1 (L1) Trigger and the High Level Trigger (HLT), is designed to make a fast selection, achieving a $10^{5}$ event rate reduction. The recent upgrade of the Level-1 trigger architecture [1][2] with improved performance allows complex correlations among trigger objects to be computed [3] and its potential can be exploited to design specific analysis-targeted algorithms. This paper describes the trigger strategy of the first dedicated Vector Boson Fusion (VBF) algorithm, designed for Higgs bosonlike analyses such as Standard Model $\mathrm{H} \rightarrow \tau \tau$. Its performance is shown and compared with the previously default trigger for that particular channel.

\section{The Vector Boson Fusion process}

The Vector Boson Fusion is an important Higgs boson production process: besides its cross section $(\sim 4 \mathrm{pb})$ being one order of magnitude lower than that of the gluon-gluon fusion process $(\sim 50 \mathrm{pb})$, its topology allows regions of phase-space with excellent signal to background ratio to be probed. Within the proton-proton interaction, the involved quarks can radiate vector bosons (Z/W) and a Higgs boson can result from their fusion. The most important characteristic of the VBF signature consists in the jets produced in the process: despite the emission of the vector bosons, the incoming quarks usually only lose a very small amount of their longitudinal energies and the energetic outgoing quarks hadronize producing jets $\left(p_{\mathrm{T}} \sim m_{H} / 2\right)$ in the forward regions of the detector. Thus the invariant mass of the two final state jets produced is large, as well as their angular separation. Since the category of events identified as produced through the VBF process is one of the most sensitive categories for the analysis of the Standard Model Higgs boson decay into in a pair of two $\tau$ leptons, exploiting the characteristic signature of the VBF process at the trigger level brings a large event yield for this selection.

\section{The VBF L1 trigger strategy}

The L1 trigger implements a sophisticated hadronic $\tau$ algorithm [4]. The $\mathrm{H} \rightarrow \tau \tau$ analysis currently relies on the Double Tau L1 trigger, to which a $14 \mathrm{kHz}$ bandwidth is allocated. It selects events with a pair of $\tau$ leptons with $p_{\mathrm{T}}>32 \mathrm{GeV}$ and $|\eta|<2.1$ targeting, as the classic trigger strategies, the Higgs decay products. In the case of the VBF trigger, the selection is specific to the production mode, hence it can be used as a complement of the Double Tau trigger in order to have a more efficient physics selection. For an event to pass the VBF L1 trigger selection, it is required to have:

- at least one jet with transverse energy $E_{\mathrm{T}}>X$; 

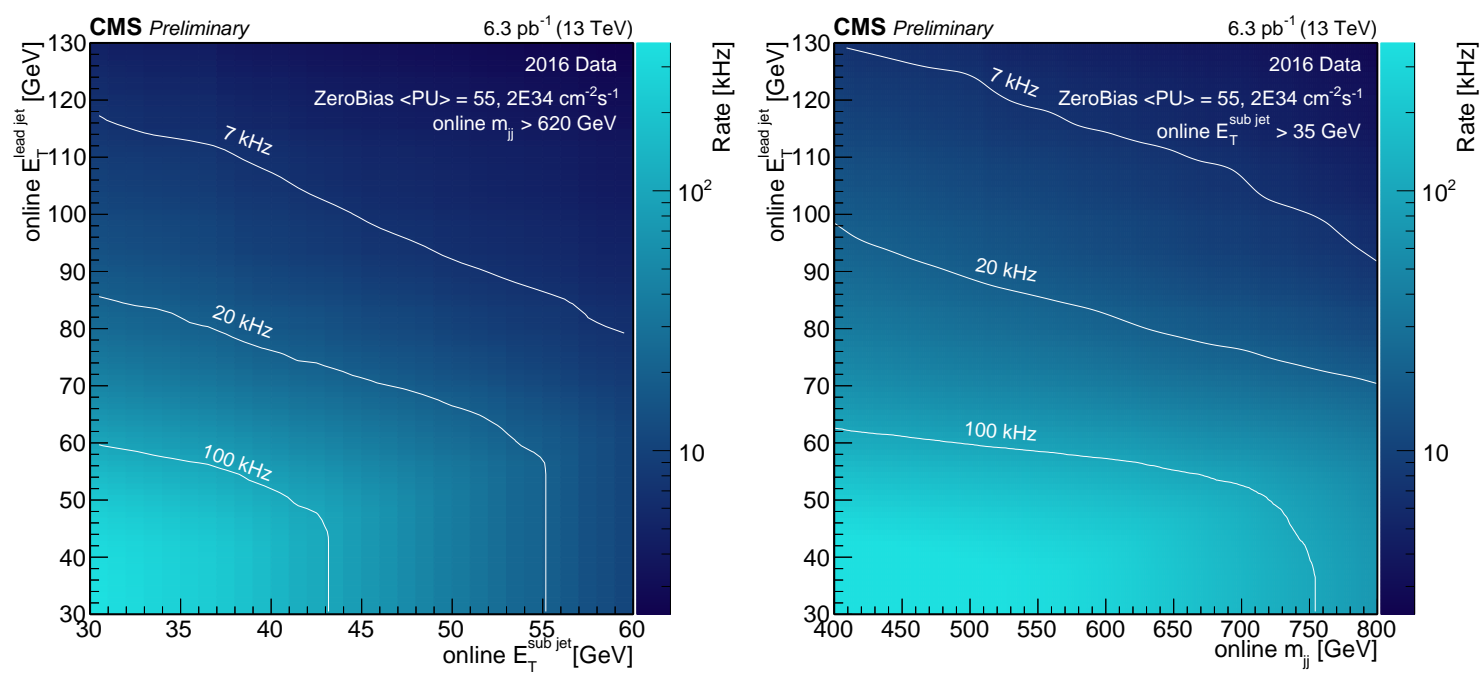

Figure 1: Rate evaluated on 2016 data and scaled to 2017 conditions for the L1 VBF trigger as a function of $X$ and $Y$ threshold with fixed $Z$ (left) as a function of $X$ and $Z$ threshold with fixed $Y$ (right) [5]. The white lines indicate the sets of thresholds for which the rate has equal values (100, $20,7 \mathrm{kHz})$.

- at least two jets with transverse energy $E_{\mathrm{T}}>Y$;

- in the collection of jets with $E_{\mathrm{T}}>Y$, at least a pair with invariant mass $m_{\mathrm{jj}}>Z$.

Two factors of merit are used to choose the $X, Y, Z$ thresholds: they should provide a high rate reduction and simultaneously a high efficiency. In particular, the thresholds are tuned in order to provide a large reduction of the additional rate with respect to the rest of the L1 selection while keeping a large gain compared to the event yield of the Double Tau trigger alone. The most promising VBF trigger selection is achieved with the thresholds $X=110 \mathrm{GeV}, Y=35 \mathrm{GeV}$ and $Z=620 \mathrm{GeV}$, giving $7.7 \mathrm{kHz}$ total rate (Fig. 1). In order to evaluate the gain of the VBF trigger against the Double Tau trigger alone, a suitable offline selection is chosen for each trigger, with thresholds always tighter than its corresponding online selection. The goal is to reproduce realistic analysislike scenarios and compare the offline event yield of the sole Double Tau trigger case with that of the combined VBF and Double Tau trigger. The increase of the event yield is achieved by an extended coverage of the phase-space, as shown in Fig. 2. On one hand, the VBF offline selection has a high $m_{\mathrm{jj}}$ threshold $\left(m_{\mathrm{jj}}>700 \mathrm{GeV}\right)$, but the lower $m_{\mathrm{jj}}$ is covered by the Double Tau selection $\left(m_{\mathrm{jj}}>400 \mathrm{GeV}\right)$. On the other hand, as the VBF trigger does not impose selection criteria on the Higgs decay products, it allows the use of $p_{\mathrm{T}}$ offline threshold on the $\tau$ leptons $\left(p_{\mathrm{T}}^{\text {sub } \tau}>20 \mathrm{GeV}\right.$ ) to be lower than that of the Double Tau selection $\left(p_{\mathrm{T}}^{\text {sub } \tau}>35 \mathrm{GeV}\right)$. As represented in Fig. 2, the gain is evaluated from the number of events passing the trigger selections and the corresponding offline selections: the events in each of the categories "Only VBF selection" and "Only DiTau selection" are events that fired only one of the triggers and pass only the offline selection corresponding to that trigger; the events in the category "DiTau and VBF" fire both triggers and pass both offline 

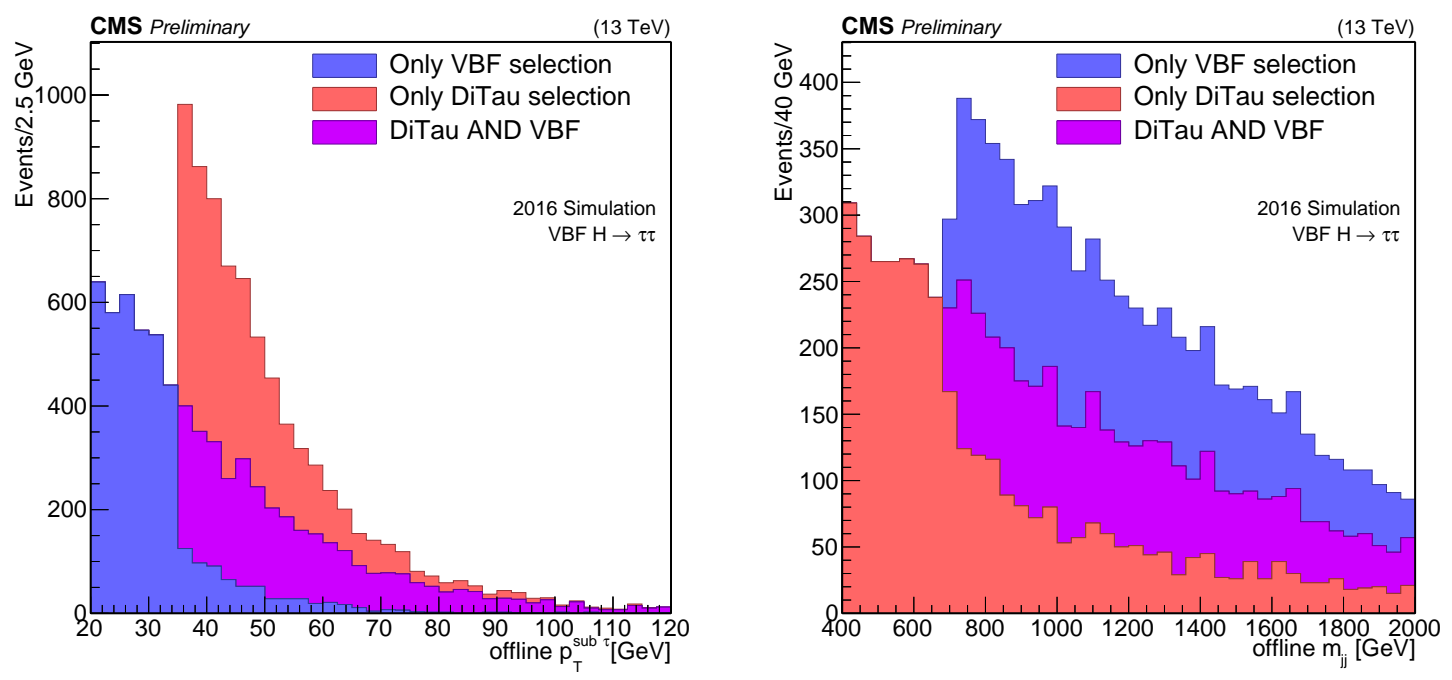

Figure 2: For $X=110 \mathrm{GeV}, Y=35 \mathrm{GeV}$ and $Z=620 \mathrm{GeV}$, event yield gain with respect to Double Tau trigger as a function of the main variables: the offline transverse momentum $p_{\mathrm{T}}^{\text {sub } \tau}$ of the subleading $\tau$ (right) and the offline invariant mass $m_{\mathrm{jj}}$ (left) [5].

selections. As mentioned above, the category "Only VBF selection" covers the region with lower $p_{\mathrm{T}}^{s u b \tau}$ and this is the main source of event yield gain. This is estimated as $N_{\text {OnlyVBF}} / N_{\text {DiTau }}=58 \%$.

\section{Conclusion}

The VBF trigger exploits the presence of two high-invariant mass jets in order to enhance the acceptance on the Higgs signal here used as a benchmark. The combination of the dedicated VBF trigger with the Double Tau trigger allows to reduce significantly the rate while keeping low $p_{\mathrm{T}}$ thresholds on the Higgs boson decay products. The most promising selection, which accepts events featuring at least two jets with $E_{\mathrm{T}}>35 \mathrm{GeV}$, a jet-jet invariant mass $m_{\mathrm{jj}}>620 \mathrm{GeV}$ and the leading jet with $E_{\mathrm{T}}>110 \mathrm{GeV}$, is included in the L1 selection for the 2017 data taking corresponding to a rate of $7.7 \mathrm{kHz}$. This trigger is meant to be a complement of the classic $\mathrm{L} 1$ triggers targeting the decay mode of the Higgs boson. For example, the Higgs boson decay channel into a pair of two $\tau$ leptons can rely both on the VBF trigger and the $\tau$ pair trigger, so that a better signal efficiency is reached for one of the most sensitive categories already at the first level of trigger selection. Adding the described VBF trigger to the currently used $\tau$ pair trigger brings $\sim 58 \%$ event yield gain for selected VBF $\mathrm{H} \rightarrow \tau \tau$ events with respect to the sole $\tau$ pair trigger. Moreover, the VBF trigger is also very interesting for other Higgs decay channels, in particular invisible $\mathrm{H}$, which can only rely on selections based on production mode topologies. The design of other analysis-targeted L1 selections, which can rely on the outstanding capabilities of the CMS L1 trigger system in building complex correlations among objects [3], will be studied in the coming months. 


\section{References}

[1] CMS Technical Design Report for the Level-1 Trigger Upgrade, CMS Collaboration (CERN-LHCC-2013-011)

[2] The CMS Level-1 Calorimeter Trigger for LHC Run II, A. Zabi (LLR, École polytechnique) for the CMS Collaboration, proceedings of the TWEPP16 International Conference in Karlsruhe 26th-30th September 2016, JINST 12 (2017) C01065 (CMS-CR-2016-360), doi:10.1088/1748-0221/12/01/C01065

[3] Data analysis at CMS Level-1 Trigger level: Migrating complex selection algorithms from offline analysis and High-Level trigger to the trigger electronics, C. Wulz (Austrian Academy of Sciences) for the CMS Collaboration, these proceedings PoS EPS-HEP2017

[4] The CMS Level-1 Tau algorithm for the LHC Run II, L. Cadamuro (LLR, École polytechnique) for the CMS Collaboration, PoS EPS-HEP2015 (CMS-CR-2015-214)

[5] Level 1 Tau trigger performance in 2016 data and VBF seeds at Level 1 trigger, CMS Collaboration (CMS DP-2017/022) 